\title{
3D-Web-GIS RFID Location Sensing System for Construction Objects
}

\author{
Chien-Ho Ko \\ Department of Civil Engineering, National Pingtung University of Science and Technology, 1 Shuefu Road, Neipu, Pingtung 912, Taiwan \\ Correspondence should be addressed to Chien-Ho Ko; fpecount@yahoo.com.tw
}

Received 10 May 2013; Accepted 9 June 2013

Academic Editors: J. Mander and B. Uy

Copyright (C) 2013 Chien-Ho Ko. This is an open access article distributed under the Creative Commons Attribution License, which permits unrestricted use, distribution, and reproduction in any medium, provided the original work is properly cited.

Construction site managers could benefit from being able to visualize on-site construction objects. Radio frequency identification (RFID) technology has been shown to improve the efficiency of construction object management. The objective of this study is to develop a 3D-Web-GIS RFID location sensing system for construction objects. An RFID 3D location sensing algorithm combining Simulated Annealing (SA) and a gradient descent method is proposed to determine target object location. In the algorithm, SA is used to stabilize the search process and the gradient descent method is used to reduce errors. The locations of the analyzed objects are visualized using the 3D-Web-GIS system. A real construction site is used to validate the applicability of the proposed method, with results indicating that the proposed approach can provide faster, more accurate, and more stable 3D positioning results than other location sensing algorithms. The proposed system allows construction managers to better understand worksite status, thus enhancing managerial efficiency.

\section{Introduction}

The construction industry is characterized by intensive manual labor and is prone to errors [1], creating significant challenges for providing a clear understanding of construction site activity [2]. A perennial issue facing construction site managers is object positioning including assets, personnel, material, and equipment [3-6]. Several attempts have been made to facilitate the location of objects on construction sites. Meade and Chignell [7] used ground penetrating radar to locate buried piping without excavation. Grau [8] presented an epistemic model based on belief functions to monitor the positions of mobile sensing nodes. His research demonstrates that the epistemic functions can correctly filter location uncertainties and effectively monitor the movements of mobile sensing nodes. Song et al. [9] tracked construction material to improve project performance and reduce the effort needed to derive project performance indicators. In their approach, materials are fitted with radio frequency identification (RFID) tags to allow for automatic identification and tracking on construction sites. Razavi and Moselhi [10] developed a construction equipment and supply location system using passive RFID tags. Skibniewski and Jang [11] introduced an architecture for construction asset tracking using wireless sensor modules to track objects via the time-offlight method. Shahi et al. [12] presented an Ultra Wide Band positioning system as a material and activity tracking tool for indoor construction projects. Global Positioning System (GPS) is frequently used for tracking objects outdoors. Pradhananga and Teizer [13] used GPS devices to automate the assessment of construction site equipment operations by continuously logging time-stamped equipment locations for analysis.

Given the alternatives available for object positioning in construction sites, Jiang et al. [14] and Nasir et al. [15] suggested methodologies for selecting appropriate technologies for various types of projects and objects and suggested that RFID is an appropriate solution for object positioning in indoor construction sites. Razavi and Moselhi [10] also demonstrated the potential for RFID as a method for object tracking in indoor construction sites.

Accurate object positioning offers the possibility of improved object visibility. However, previous studies have either treated positioning algorithms as separate from display systems or have tended to use one- or two-dimensional maps 
to indicate object locations, thus obscuring object visibility. Displaying objects in three-dimensional (3D) space requires a corresponding location sensing algorithm. Ko [16] proposed an RFID 3D location sensing algorithm, but the algorithm had trouble deriving smooth convergences while searching for the target objects.

The present study develops a 3D-Web-GIS RFID location sensing system to locate objects in indoor construction sites. An improved RFID 3D location sensing algorithm is established, combining Simulated Annealing (SA) and the gradient descent method to overcome the convergence problem while locating objects. The 3D-Web-GIS RFID location sensing system manipulates the location sensing algorithm to better relate the display of construction objects to the real world and help managers better understand construction site activity. This study begins by introducing indoor sensing networks and then explains the evolutionary process of the proposed RFID 3D location sensing algorithm. Section four describes the development of the 3D-Web-GIS RFID location sensing system. Section five describes a demonstration of the system on a construction site. Finally, the paper concludes with suggestions for future research directions.

\section{Indoor Sensing Networks}

Construction sites require both indoor and outdoor sensing $[17,18]$, but these sensing contexts require different types of networks and technologies [19]. GPS, a relatively mature location sensing technique, is frequently used in outdoor construction sites [20], but indoor location remains a challenge. This study thus focuses on improving the accuracy and efficiency of the location of indoor construction objects including material, equipment, personnel, and machinery. Construction site activity can only be understood through the simultaneous tracking of multiple objects. In a passive location system, RFID antennas are distributed at reference coordinates within the location space [21], and the target objects are fitted with passive RFID tags [22]. This provides a relatively low-cost solution as compared to active RFID systems in which the target objects have to be equipped with antennas. A passive location mode that attaches an active RFID tag on the target object [23] is therefore selected.

The indoor sensing networks are constructed using RFID readers and tags, as shown in Figure 1. An active RFID system was used to expand the sensing space. Four RFID antennas were set at four corners of the hexahedron space. Nine reference tags were uniformly distributed in the space to build a location sensing network. Through the network topology, signal strengths of the RFID tags from different directions with diverse distances can be collected. The collected signal strength was then analyzed using a 3D location sensing algorithm to calculate target object locations.

\section{Location Sensing Algorithms}

3.1. Location Concept. This research develops an RFID 3D location sensing algorithm using a trilateration method [2426] that calculates the target object location using distances

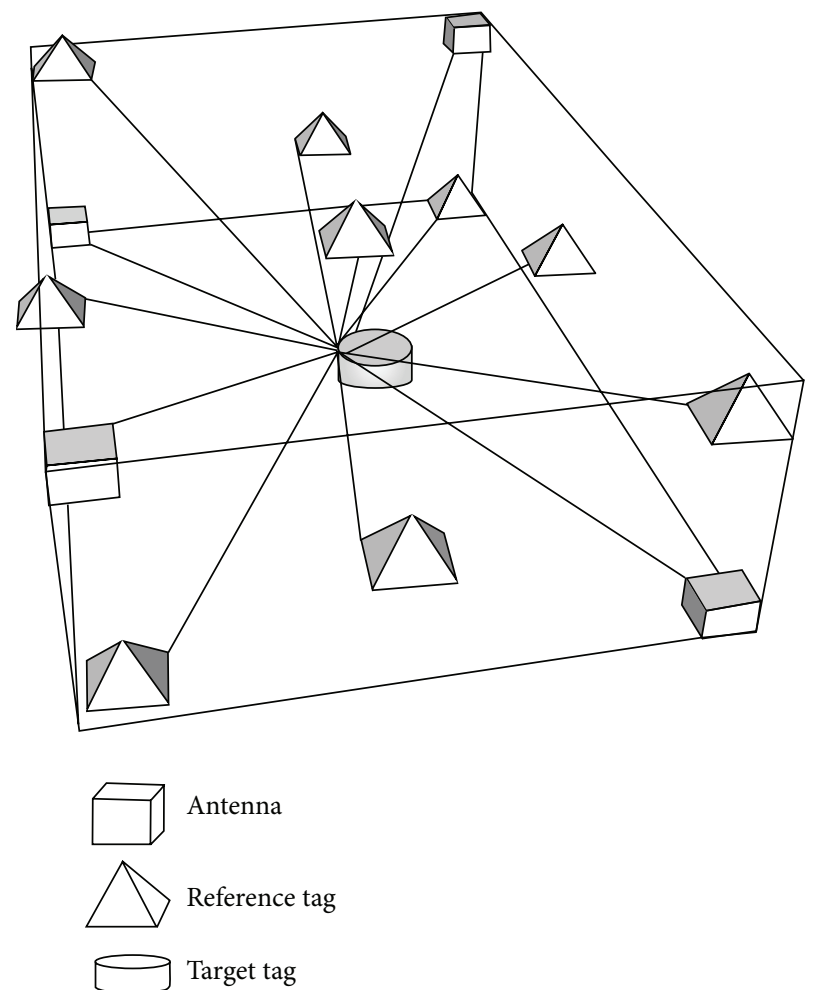

FIGURE 1: Indoor sensing networks.

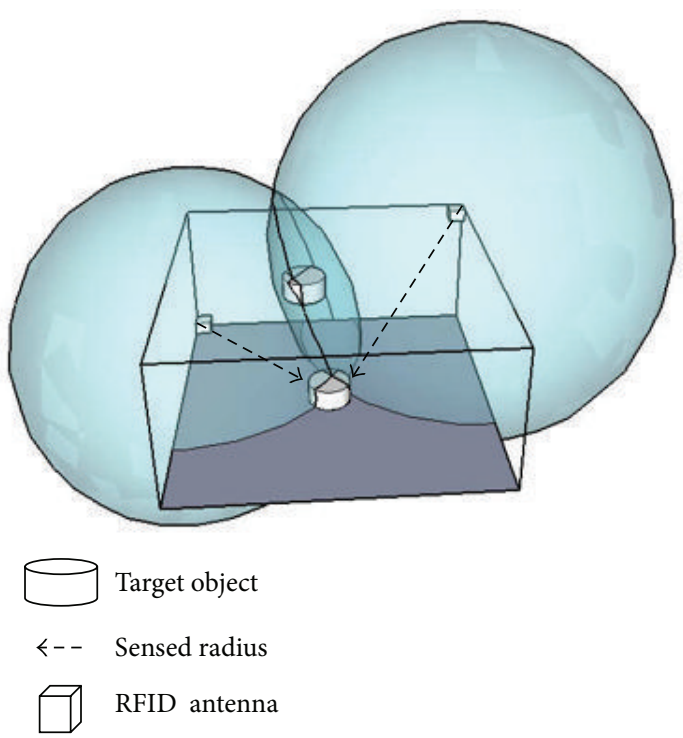

FIGURE 2: 3D trilateration location concept.

from the RFID antennas to the target object. In a 3D space, a single RFID antenna can sense its distance to an RFID tag using Received Signal Strength Indication (RSSI). The possible location of the target object could be expressed as a sphere with a radius of the sensed distance. Adding a second antenna, the solution space is an intersection of the two spheres with the two sensed radii, as shown in Figure 2. Using the same concept, the target object location could be further 
narrowed to few points using three antennas. Four antennas can be expected to produce a highly specific location.

3.2. Location Algorithm. Ko [16] developed an RFID 3D location sensing algorithm using the gradient descent method. However, that method used fixed adjustment coefficients to search for the target object location. In a large space, the adjustment coefficient has to be increased to reduce the amount of computational time required, but this may make convergence difficult. A small space, on the other hand, needs smaller adjustment coefficients to converge. Selection of the adjustment coefficients appropriate for the dimensions of the given search space is achieved by trial and error. Simulated Annealing (SA), a technique analogizing the annealing of metals for stable global search [27], could potentially solve this problem. This study thus hybridizes SA and gradient descent methods to locate construction-related objects in a 3D space. The improved RFID 3D location sensing algorithm is shown and explained in Figure 3.

3.2.1. Initializing Location. The first step of the algorithm is to initiate a location search for the target object. The location of target object $i$ in 3D space is noted as $\left(x_{i}, y_{i}, z_{i}\right)$.

3.2.2. Sensing Distances. The trilateration location method requires the distances from each antenna to the target object. The proposed method senses the distance using RSSI, with an example shown in Figure 4. An antenna receives a signal with a given strength level from the active RFID tag attached to the target object. Through the RSSI curve, the received signal strength can be converted to a distance.

3.2.3. Calculating Error. This step calculates a positioning error between the initial location and the sensed location, which will be used to adjust the target object location in the next step. The positioning error of target object $i$ and antenna $k\left(e_{i k}\right)$ is calculated using the following equation:

$$
e_{i k}=\left(S_{i k}-\bar{S}_{i k}\right)
$$

where $S_{i k}$ is a sensed distance between target object $i$ and antenna $k$ converted using RSSI; $\bar{S}_{i k}$ is the distance in $3 \mathrm{D}$ space between target object $i$ at $\left(x_{i}, y_{i}, z_{i}\right)$ and antenna $k$ at $\left(x_{k}, y_{k}, z_{k}\right)$ calculated using the following equation:

$$
\bar{S}_{i k}=\sqrt{\left(x_{i}-x_{k}\right)^{2}+\left(y_{i}-y_{k}\right)^{2}+\left(z_{i}-z_{k}\right)^{2}} .
$$

3.2.4. Refining Coordinates. SA is combined with the gradient descent method to narrow the potential location of the target object. SA is used to gradually decrease the adjustment (i.e., cooling down) to help the algorithm converge, while the gradient descent method is used to reduce location error.

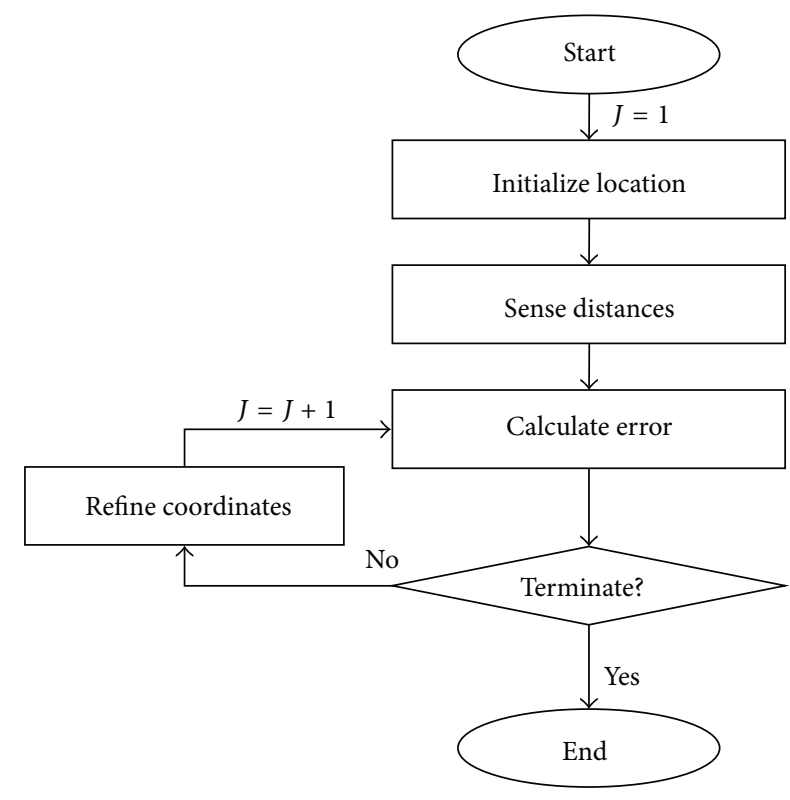

FIGURE 3: RFID 3D location sensing algorithm.

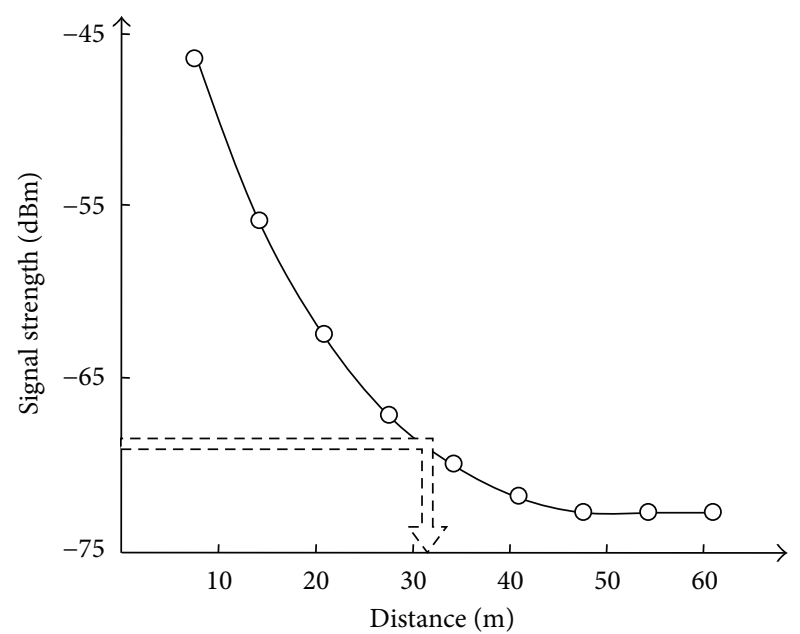

FIGURE 4: Received signal strength indication.

The target object's location at the epoch $(j)$ is adjusted using the following equation:

$$
\begin{aligned}
& \left(x_{i}(j+1), y_{i}(j+1), z_{i}(j+1)\right) \\
& \quad=\left\{\begin{array}{l}
x_{i}(j+1)=x_{i}(j)+\Delta x_{i}(j) \\
y_{i}(j+1)=y_{i}(j)+\Delta y_{i}(j) \\
z_{i}(j+1)=z_{i}(j)+\Delta z_{i}(j),
\end{array}\right.
\end{aligned}
$$

where $\left(\Delta x_{i}(j), \Delta y_{i}(j), \Delta z_{i}(j)\right)$ is the amount of adjustment for the $x_{-}, y_{-}$, and $z$-axes. SA is applied to cool down the adjustment, as shown in the following equation:

$$
\left(\Delta x_{i}(j), \Delta y_{i}(j), \Delta z_{i}(j)\right)=\left\{\begin{array}{l}
\Delta x_{i}(j)=\alpha_{x} \beta_{x} \gamma_{x} \\
\Delta y_{i}(j)=\alpha_{y} \beta_{y} \gamma_{y} \\
\Delta z_{i}(j)=\alpha_{z} \beta_{z} \gamma_{z}
\end{array}\right.
$$


where $\left(\alpha_{x}, \alpha_{y}, \alpha_{z}\right)$ is adjustment rate for the $x$-, $y$-, and $z$-axes; $\left(\beta_{x}, \beta_{y}, \beta_{z}\right)$ represents the temperature formulated in (5); $\left(\gamma_{x}, \gamma_{y}, \gamma_{z}\right)$ is the cooling speed represented in (6). Consider

$$
\left(\beta_{x}, \beta_{y}, \beta_{z}\right)=\left\{\begin{array}{l}
\beta_{x}=\frac{\left(S_{i k}-\bar{S}_{i k}\right)}{\left|S_{i k}-\bar{S}_{i k}\right|} \exp \left(\frac{-1}{\alpha_{x}\left|x_{i} \delta_{i k}\right|}\right), \\
\beta_{y}=\frac{\left(S_{i k}-\bar{S}_{i k}\right)}{\left|S_{i k}-\bar{S}_{i k}\right|} \exp \left(\frac{-1}{\alpha_{y}\left|y_{i} \delta_{i k}\right|}\right), \\
\beta_{z}=\frac{\left(S_{i k}-\bar{S}_{i k}\right)}{\left|S_{i k}-\bar{S}_{i k}\right|} \exp \left(\frac{-1}{\alpha_{z}\left|z_{i} \delta_{i k}\right|}\right), \\
\left(\gamma_{x}, \gamma_{y}, \gamma_{z}\right)=\exp (\mu) .
\end{array}\right.
$$

In (5), the $k$ RFID antenna gradient for target $i\left(\delta_{i k}\right)$ can be calculated using (7). The $\mu$ shown in (6) is a parameter simulating the cooling process, which is formulated using (8). Consider

$$
\begin{gathered}
\delta_{i k}=\bar{s}_{i k} \times e_{i k}, \\
\mu=-0.2 \times(j-1) .
\end{gathered}
$$

The $k$ RFID antenna adjusts the target object's location using (3) to (8). The error of the target object $i$ for $m$ antennas $\left(\varepsilon_{i}\right)$ is calculated using the Root Mean Square Error (RMSE), as shown in the following equation:

$$
\varepsilon_{i}=\sqrt{\frac{\sum_{k=1}^{m}\left(\left(S_{i k}-\bar{S}_{i k}\right) / S_{i k}\right)^{2}}{m}} .
$$

3.2.5. Terminating Conditions. The algorithm locates the target object's location using an iterative adjustment process. The termination conditions can be met if epoch number $(j)$ reaches a predetermined criterion and/or the $\operatorname{RMSE}\left(\varepsilon_{i}\right)$ is smaller than an assigned number. The predetermined epoch number can ensure that the location algorithm is completed within a specified duration, while the preassigned RMSE ensures the location's accuracy.

\section{System Development}

4.1. Use Case. The Rational Unified Process (RUP) [28] and Unified Modeling Language (UML) [29] were used to develop the 3D-Web-GIS RFID location sensing system. To identify system requirements, Use Case, which is regarded as a highlevel system descriptor, is used for system analysis and design. Figure 5 shows the Use Case diagram of the 3D-Web-GIS RFID location sensing system. The diagram shows how the system can be used by construction managers to locate objects in construction sites and by project stakeholders to easily understand the system.

The Use Case used in this study is explained as follows:

(i) Use Case: 3D positioning,

(ii) actor: construction managers,

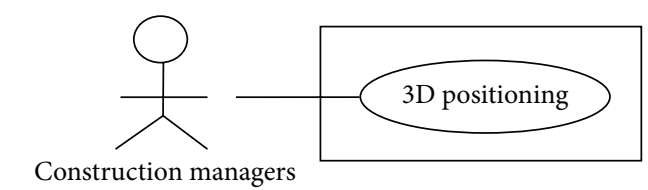

Figure 5: Use Case diagram.

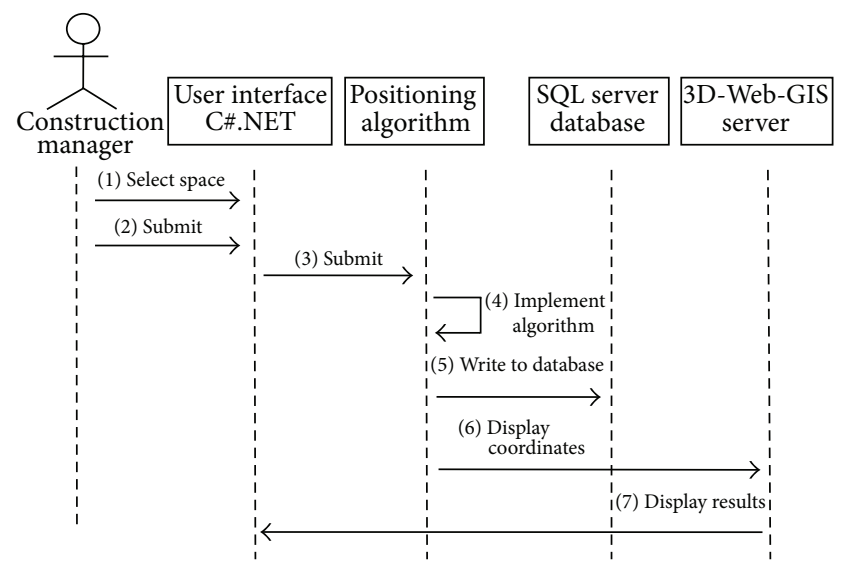

FIGURE 6: Positioning sequence diagram.

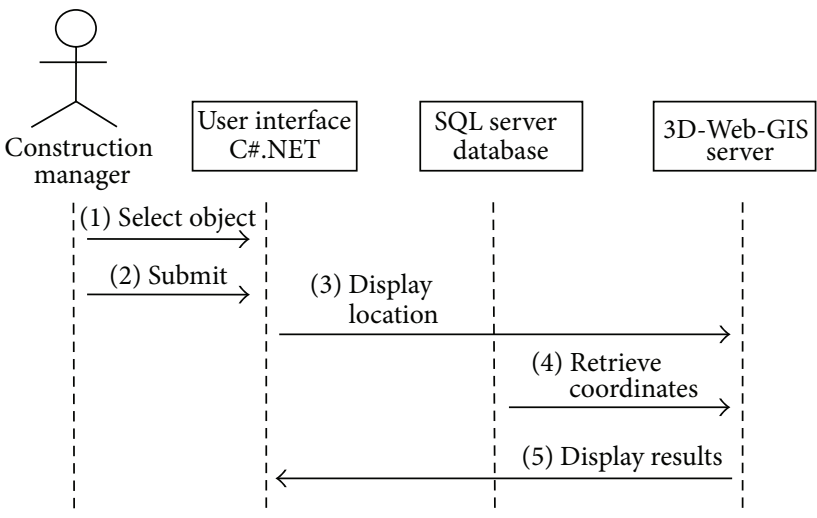

FIGURE 7: Browsing sequence diagram.

(iii) type: primary,

(iv) Descriptions:

(1) users select an object, and the system then displays the object's location in $3 \mathrm{D}$ space,

(2) users click the displayed object to retrieve information about the object.

The Use Case identifies two system functions: position and browse. These functions are explained in Table 1, while their sequence diagrams are shown in Figures 6 and 7.

4.2. System Architecture. Figure 8 displays the architecture of the 3D-Web-GIS RFID location sensing system in three tiers. Location sensing algorithm parameters and object coordinates are stored in the storage layer. The $3 \mathrm{D}$ positioning algorithm is in the application logic layer that implements system 
TABLE 1: Location sensing system functions.

\begin{tabular}{lll}
\hline Function & Explanation & Category \\
\hline Position & $\begin{array}{l}\text { Users select a construction site and space. The system calculates and displays object locations } \\
\text { using the 3D-Web-GIS system. }\end{array}$ & $\begin{array}{l}\text { Users select a construction-related object. The system displays information about the selected } \\
\text { object using the 3D-Web-GIS system. }\end{array}$ \\
\hline Browse & Evident \\
\hline
\end{tabular}

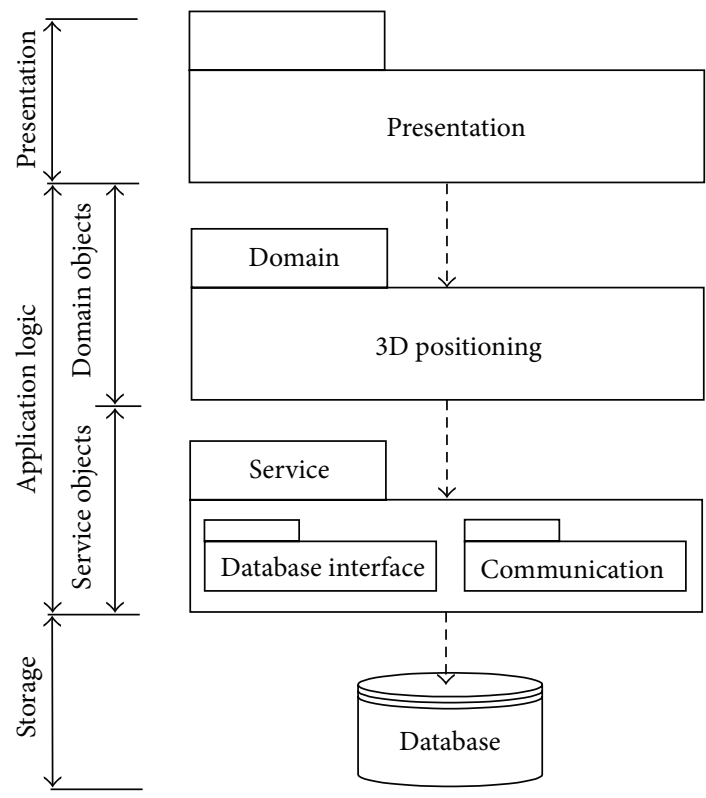

Figure 8: System architecture.

functions. The presentation layer provides user interfaces allowing users to interact with the application logic layer. The system was developed using Microsoft Visual Studio. Net (C\#.NET) with SQL server database. Construction object geographic information is displayed by integrating an ESRI ArcGIS Server with the 3D extension module via C\#.NET.

\section{Verification}

To validate its feasibility, the proposed algorithm was applied to a real construction site: a $926 \mathrm{~cm} \times 535 \mathrm{~cm} \times 211 \mathrm{~cm}$ indoor space on the third floor of a construction site. The target object is located at $(694,400,75)$. An adjustment rate of (0.5) is used for $\left(\alpha_{x}, \alpha_{y}, \alpha_{z}\right)$.

Setting the initial location of the target object at $(1,1,1)$, Figure 9 compares the convergence trend of the proposed method with that developed by Ko [16]. In the figure, $x$, $y$, and $z$ are convergence trends of the previous method, while $x_{1}, y_{1}$, and $z_{1}$ are those of the proposed method. The proposed method locates the target object at iteration 60 , as opposed to iteration 90 for the previous method. The previous method adjusts the target object's location using gradient decent method. Thus, although adjustments move in the right direction, the convergence becomes spiky in later stages. By contrast, the proposed method combines SA and the gradient decent method to adjust the target object's location.

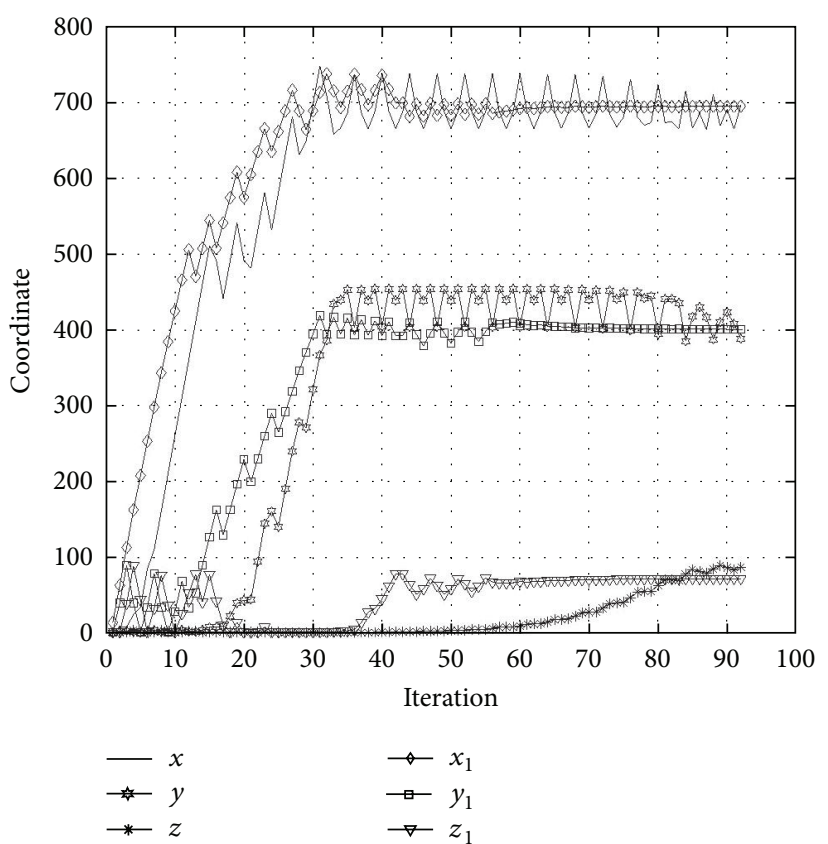

FIGURE 9: Location convergence comparison.

In the early stages of positioning, both methods display the same conspicuous adjustments. In the cooling down stage, smoother adjustments are applied to locate the target object. Figure 10 compares the error convergence between the two methods. Figure 11 shows the locus of the two methods in 3D space while positioning the target object. As discussed, the proposed method locates the target object faster, more accurately, and more stably in 3D space. Finally, the location of the target object is displayed using the developed 3D-WebGIS system, as shown in Figure 12, and the location of the construction objects can be visualized. By clicking the located objects, object information is retrieved from the database, as shown in Figure 13.

\section{Conclusions}

This study hybridizes SA and the gradient descent method to develop a 3D RFID location sensing algorithm. A 3D-WebGIS system is developed to run the algorithm and display the location of target objects. The proposed method is validated by application to a real construction site, with performance comparisons to the previous best method.

In the proposed algorithm, SA is used to stabilize the search process while the gradient descent method is used to 


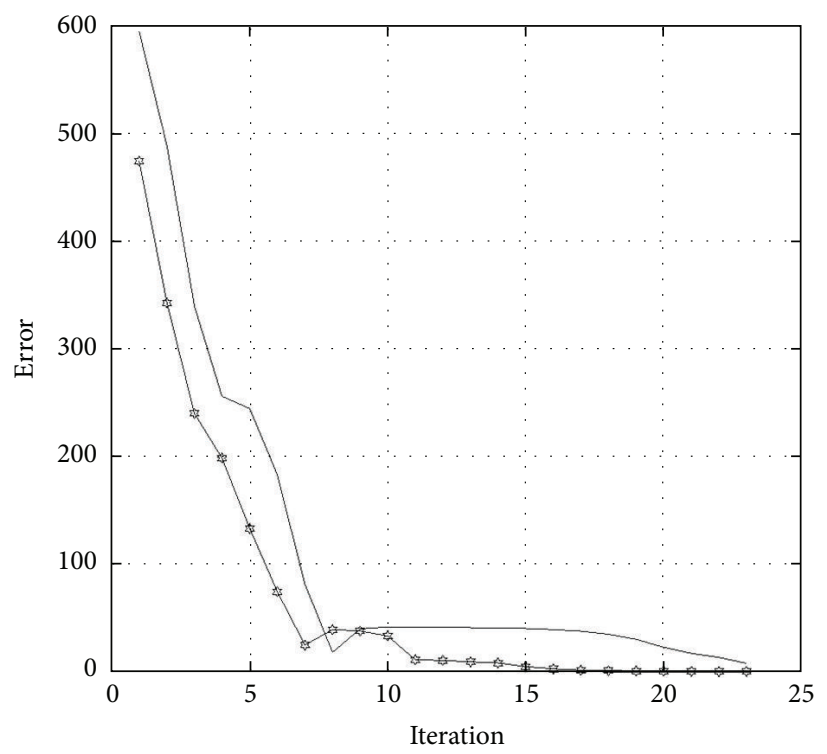

Previous

* Proposed

FIGURE 10: Error convergence comparison.

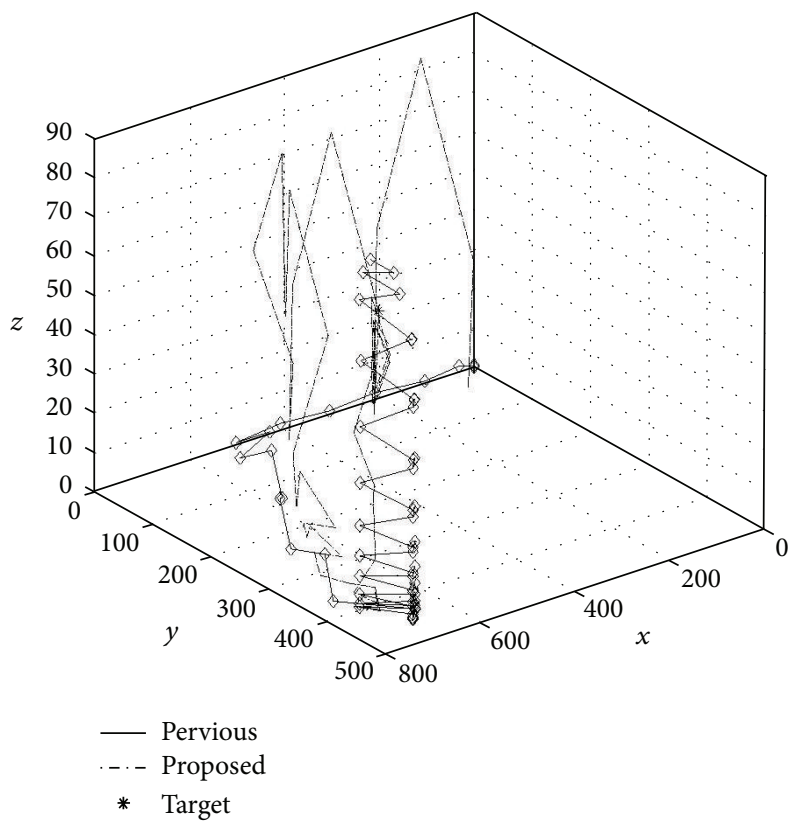

FIgURE 11: 3D locus comparison.

increase location accuracy. At the beginning of the search, while the temperature is high, large location adjustments are made, thus saving time for positioning. The temperature gradually cools as the positioning process enhances the convergence. Application to a real construction site validates that combining SA with the gradient descent method improves the speed, accuracy, and stability of results over those obtained using the previous location sensing algorithm in $3 \mathrm{D}$ positioning. Furthermore, the previous $3 \mathrm{D}$ positioning algorithm needs to determine algorithm parameters according to location space dimension. The proposed positioning algorithm, however, frontloads target object searching to the beginning of the search process and gradually cools down over time and thus may not need to predetermine algorithm parameters due to the size of the spatial dimensions.

The developed 3D-Web-GIS RFID location sensing system can be accessed through the Internet. Positioning results are visualized in $3 \mathrm{D}$ environment, and users can browse information related to the located construction objects. The system allows construction managers to locate construction objects at any time, from anywhere using any operating systems, thus enhancing managerial efficiency. 


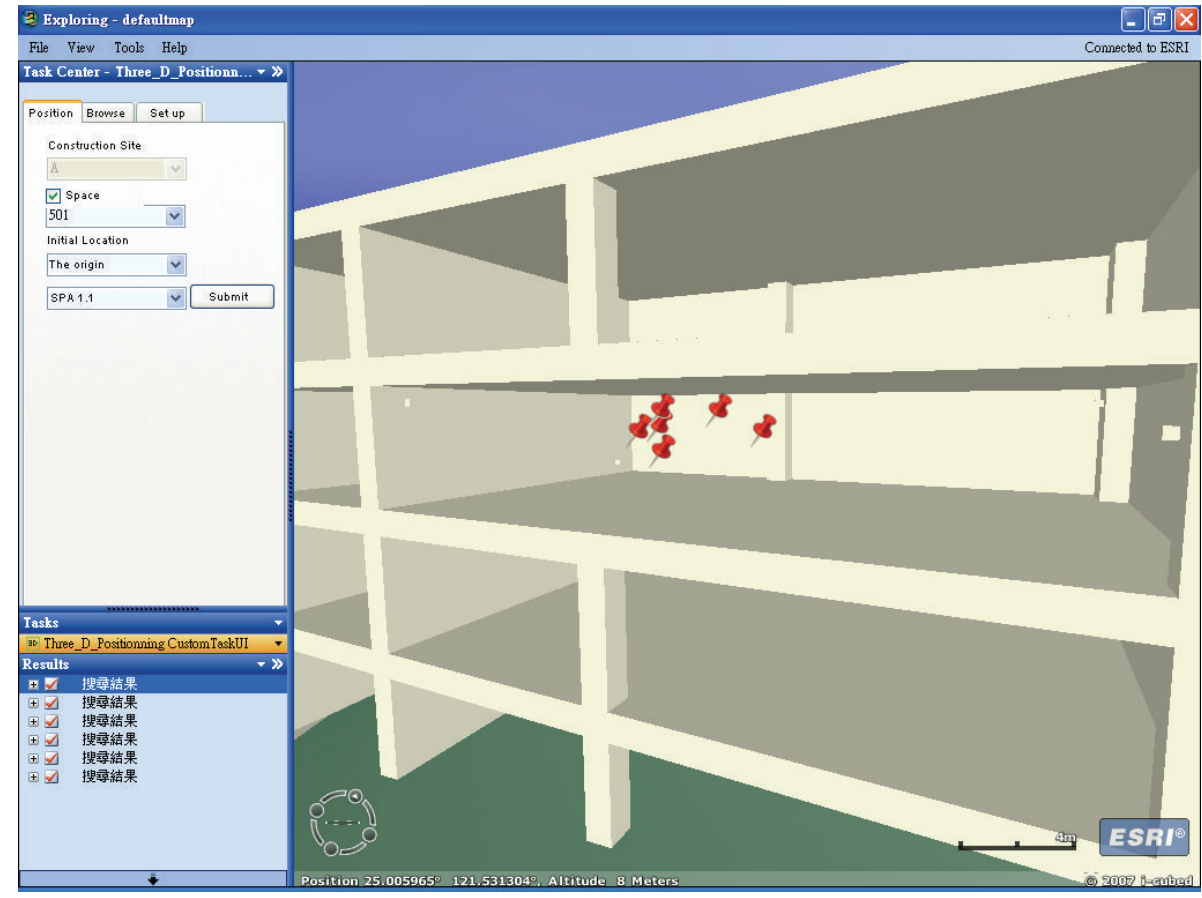

FIGURE 12: Target object location display.

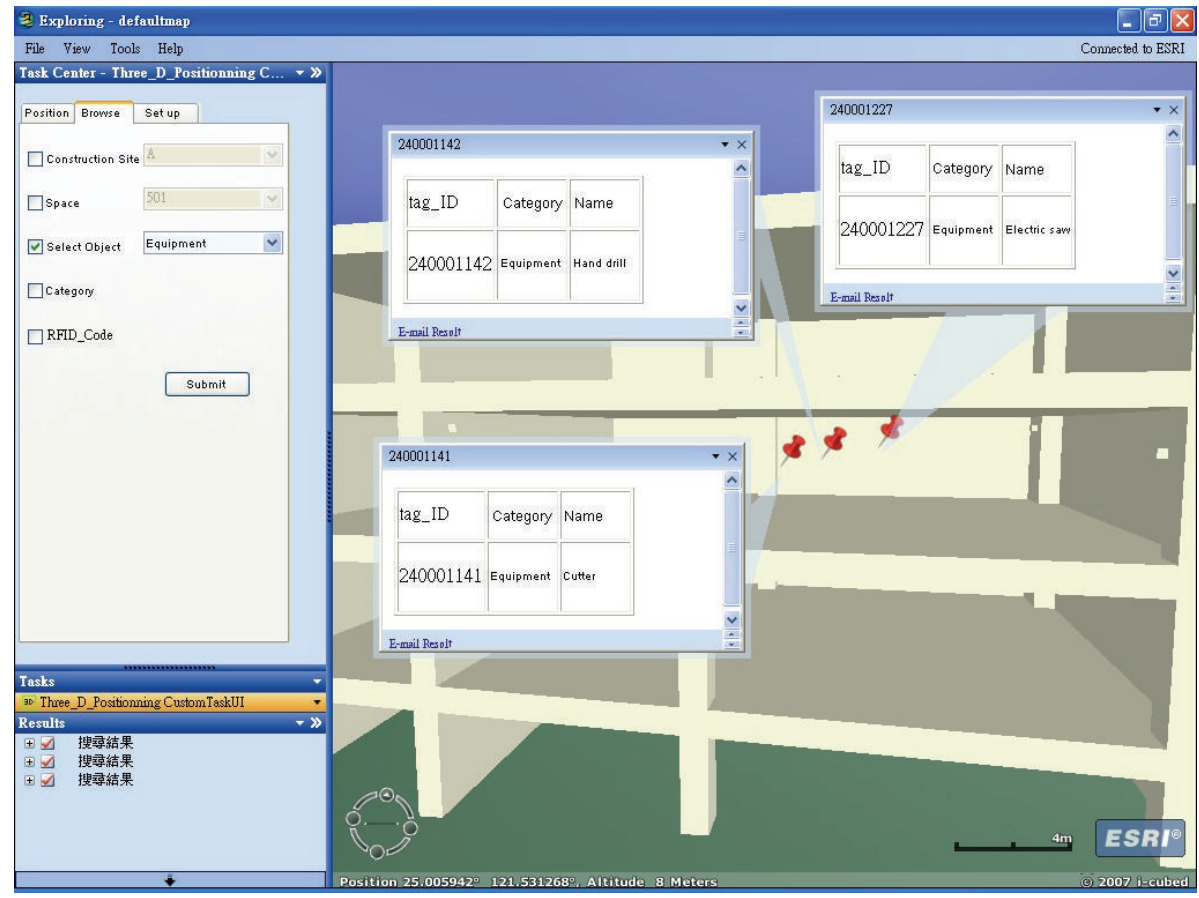

FIGURE 13: Browsing construction object information.

This study did not take into account the RFID signal attenuation effect caused by environmental factors, and future studies could modify the proposed algorithm to consider signal attenuation. Future work could also use this $3 \mathrm{D}$ location sensing technology to develop mechanisms for tracking the movement of construction objects within construction sites.

\section{Acknowledgments}

This research was funded by the National Science Council (Grant no. NSC 99-2622-E-020-006-CC3) and Architecture and Building Research Institute (Taiwan), whose supports are gratefully acknowledged. Any opinions, findings, conclusions, or recommendations expressed in this paper are those 
of the author and do not reflect the views of the National Science Council and Architecture and Building Research Institute. The writer would also like to thank the investigated construction site for supporting this study. Special thanks are due to research assistant Tai-Ru Hong who helped in programming.

\section{References}

[1] T. Cheng, M. Venugopal, J. Teizer, and P. A. Vela, "Performance evaluation of ultra wideband technology for construction resource location tracking in harsh environments," Automation in Construction, vol. 20, no. 8, pp. 1173-1184, 2011.

[2] R. Navon and O. Berkovich, "An automated model for materials management and control," Construction Management and Economics, vol. 24, no. 6, pp. 635-646, 2006.

[3] K. Morioka, S. Kovacs, J.-H. Lee, and P. Korondi, "A cooperative object tracking system with fuzzy-based adaptive camera selection," International Journal on Smart Sensing and Intelligent Systems, vol. 3, no. 3, pp. 338-358, 2010.

[4] S. Cook, J. Bittner, and T. Adams, "Developing an asset management tool to collect and track commitments on environmental mitigation features," Transportation Research Record, no. 2160, pp. 21-28, 2010.

[5] J. Teizer and P. A. Vela, "Personnel tracking on construction sites using video cameras," Advanced Engineering Informatics, vol. 23, no. 4, pp. 452-462, 2009.

[6] A. H. Behzadan, Z. Aziz, C. J. Anumba, and V. R. Kamat, "Ubiquitous location tracking for context-specific information delivery on construction sites," Automation in Construction, vol. 17, no. 6, pp. 737-748, 2008.

[7] R. B. Meade and R. J. Chignell, “Tool advances pipe location and construction planning," Pipeline and Gas Journal, vol. 224, no. 4, p. 3, 1997.

[8] D. Grau, "Epistemic model to monitor the position of mobile sensing nodes on construction sites with rough location data," Journal of Computing in Civil Engineering, vol. 26, no. 1, pp. 141150, 2012.

[9] J. Song, C. T. Haas, and C. H. Caldas, "Tracking the location of materials on construction job sites," Journal of Construction Engineering and Management, vol. 132, no. 9, pp. 911-918, 2006.

[10] S. N. Razavi and O. Moselhi, "GPS-less indoor construction location sensing," Automation in Construction, vol. 28, pp. 128136, 2012.

[11] M. J. Skibniewski and W.-S. Jang, "Simulation of accuracy performance for wireless sensor-based construction asset tracking," Computer-Aided Civil and Infrastructure Engineering, vol. 24, no. 5, pp. 335-345, 2009.

[12] A. Shahi, A. Aryan, J. S. West, C. T. Haas, and R. C. G. Haas, "Deterioration of UWB positioning during construction," Automation in Construction, vol. 24, pp. 72-80, 2012.

[13] N. Pradhananga and J. Teizer, "Automatic spatio-temporal analysis of construction site equipment operations using GPS data," Automation in Construction, vol. 29, pp. 107-122, 2013.

[14] S. Jiang, W. S. Jang, and M. J. Skibniewski, "Selection of wireless technology for tracking construction materials using a fuzzy decision model," Journal of Civil Engineering and Management, vol. 18, no. 1, pp. 43-59, 2012.
[15] H. Nasir, C. T. Haas, D. A. Young, S. N. Razavi, C. Caldas, and P. Goodrum, "An implementation model for automated construction materials tracking and locating," Canadian Journal of Civil Engineering, vol. 37, no. 4, pp. 588-599, 2010.

[16] C.-H. Ko, "RFID 3D location sensing algorithms," Automation in Construction, vol. 19, no. 5, pp. 588-595, 2010.

[17] X. Shen and M. Lu, "A framework for indoor construction resources tracking by applying wireless sensor networks," Canadian Journal of Civil Engineering, vol. 39, no. 9, pp. 1083-1088, 2012.

[18] Y. Zhu, X. Ding, Z. Li, and S. Zhou, "Discussion on the application of GPS using in Marine construction survey," Journal of Computers, vol. 7, no. 7, pp. 1663-1670, 2012.

[19] H. M. Khoury and V. R. Kamat, "Evaluation of position tracking technologies for user localization in indoor construction environments," Automation in Construction, vol. 18, no. 4, pp. 444$457,2009$.

[20] T.-H. Yi, H.-N. Li, and M. Gu, "Recent research and applications of GPS-based monitoring technology for high-rise structures," Structural Control and Health Monitoring, vol. 20, no. 5, pp. 649670, 2013.

[21] F. Gustafsson and F. Gunnarsson, "Mobile positioning using wireless networks: Possibilities and fundamental limitations based on available wireless network measurements," IEEE Signal Processing Magazine, vol. 22, no. 4, pp. 41-53, 2005.

[22] W. Gueaieb and S. Miah, "An intelligent mobile robot navigation technique using RFID technology," IEEE Transactions on Instrumentation and Measurement, vol. 57, no. 9, pp. 1908-1917, 2008.

[23] R. Want, A. Hopper, V. Falcao, and J. Gibbons, "Active badge location system," ACM Transactions on Information Systems, vol. 10, no. 1, pp. 91-102, 1992.

[24] T. F. Bechteler and H. Yenigün, "2-D localization and identification based on SAW ID-tags at $2.5 \mathrm{GHz}$," IEEE Transactions on Microwave Theory and Techniques, vol. 51, no. 5, pp. 1584-1590, 2003.

[25] D. E. Manolakis, "Efficient solution and performance analysis of 3-D position estimation by trilateration," IEEE Transactions on Aerospace and Electronic Systems, vol. 32, no. 4, pp. 1239-1248, 1996.

[26] F. Thomas and L. Ros, "Revisiting trilateration for robot localization," IEEE Transactions on Robotics, vol. 21, no. 1, pp. 93-101, 2005.

[27] W. H. Press, S. A. Teukolsky, W. T. Vetterling, and B. P. Flannery, The Art of Scientific Computing, Cambridge University Press, New York, NY, USA, 2007.

[28] P. Kruchten, The Rational Unified Process: An Introduction, Addison-Wesley Professional, Reading, Mass, USA, 2003.

[29] G. Booch, J. Rumbaugh, and I. Jacobson, The Unified Modeling Language User Guide, Addison-Wesley Professional, Reading, Mass, USA, 2005. 

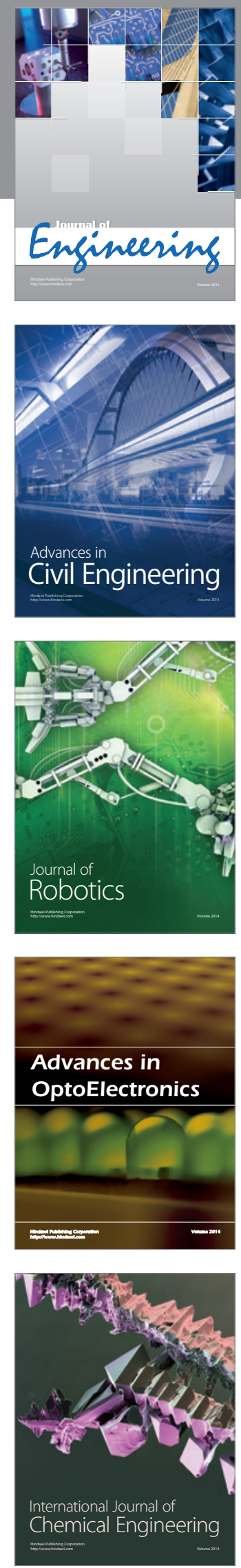

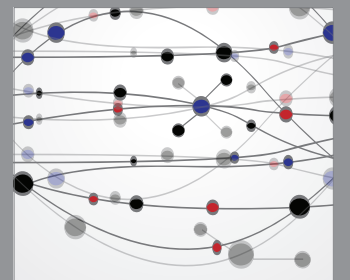

The Scientific World Journal
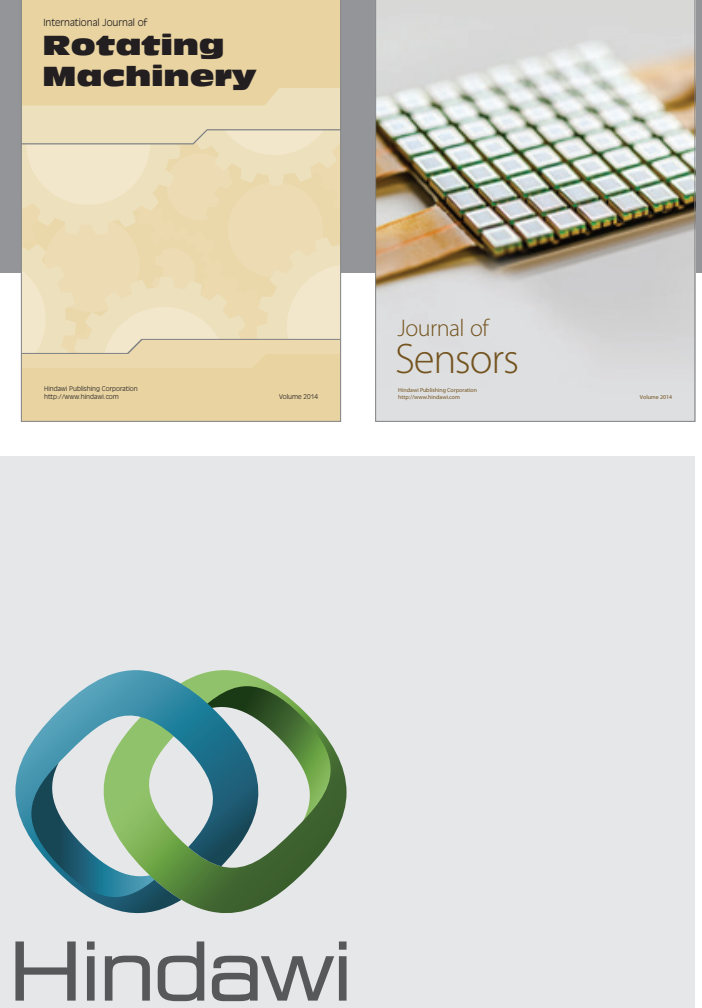

Submit your manuscripts at http://www.hindawi.com
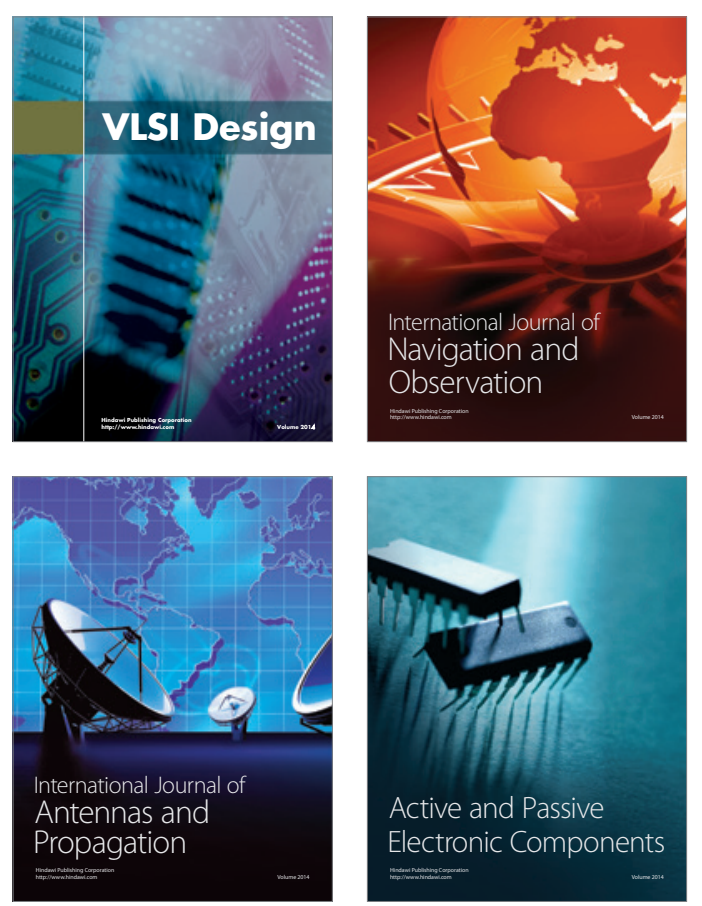
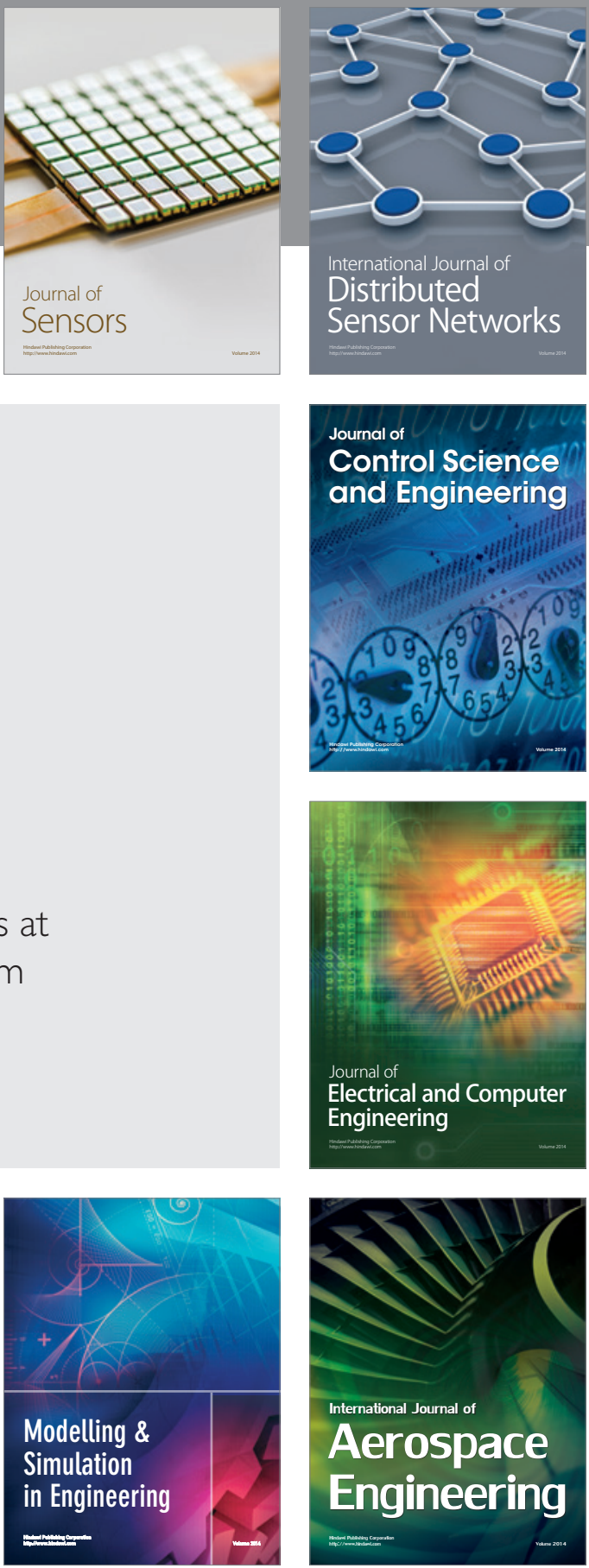

Journal of

Control Science

and Engineering
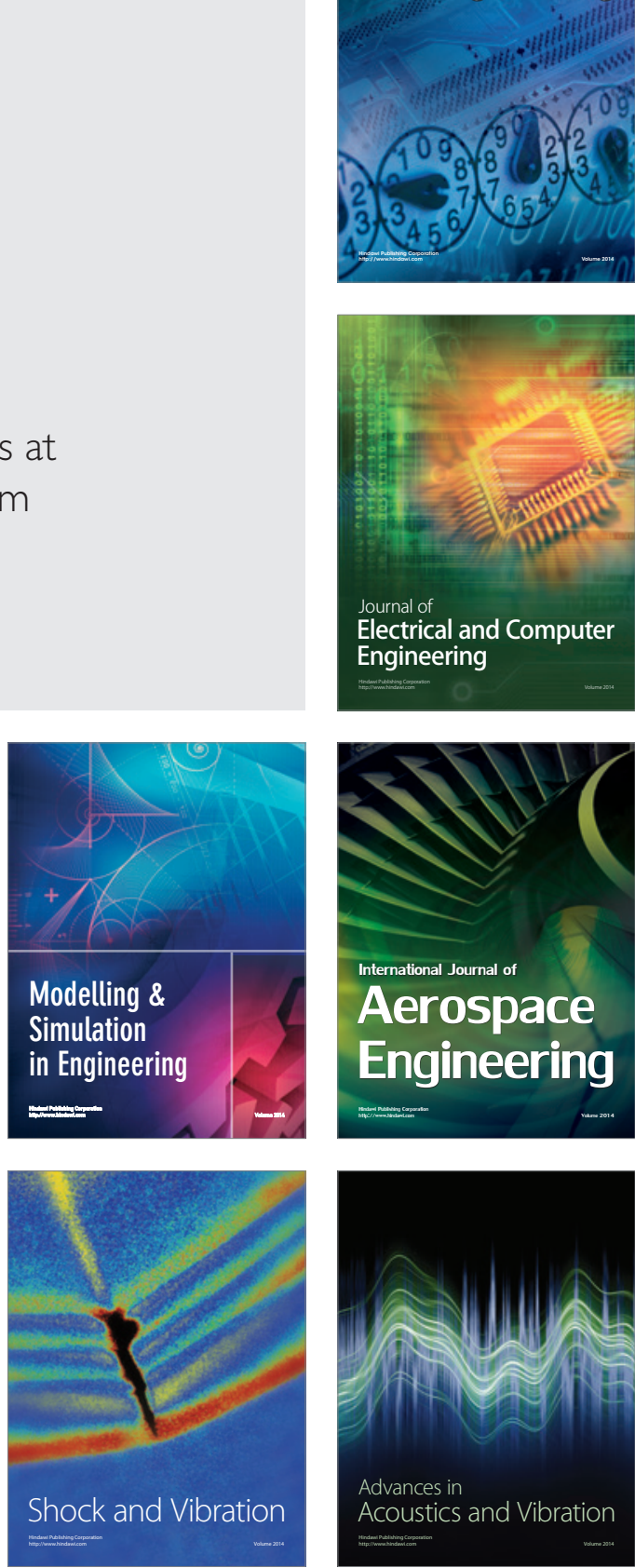